\title{
Eutanasia, suicidio asistido y principio del doble efecto. Réplica al profesor Rodolfo Figueroa
}

\author{
ALEJANDRO MIRANDA M. ${ }^{\mathrm{a}}$
}

\section{Euthanasia, assisted suicide, and the principle of double effect. A reply to Rodolfo Figueroa}

The purpose of this paper is to defend the traditional application of the principle of double effect as a criterion for assessing the permissibility of actions that have as their common aim to end the suffering of seriously ill patients. According to this principle, euthanasia and physician-assisted suicide are always illicit acts, while the same is not said for other actions that bring about patient's death as a foreseen effect, namely, palliative treatments that hasten death or failure or interruption of life support. The reason for this difference is that, in the first two cases, the patient's death is intended as a means of pain relief; whereas, in the latter two, death is only a side effect of a medical act, an act justifiable if it is necessary to achieve a proportionate good. In a recent issue of this Journal, Professor Rodolfo Figueroa denied the soundness of the principle of double effect and maintained that all actions described above should be considered equivalent in law enforcement. Here, the author presents a reply to that argument, and also offers a justification of the afore said principle's core, that is, the moral and legal relevance of the distinction between intended effects and foreseen side effects.

(Rev Med Chile 2012; 140: 261-265).

Key words: Double effect principle; Euthanasia; Suicide, assisted.



$\mathrm{R}$ odolfo Figueroa ha dirigido en estas páginas una crítica contra el uso del principio del doble efecto (PDE) como regla para evaluar la licitud de acciones que tienen por finalidad común poner término a los sufrimientos de enfermos graves ${ }^{1}$. En particular, Figueroa sostiene que la distinción que subyace al PDE -la distinción entre efectos intentados y efectos colaterales previstos (distinción intentado/colateral) - carece de relevancia moral y debe, por tanto, ser excluida del ordenamiento jurídico. En este trabajo me propongo defender dicha distinción para mostrar que el PDE constituye una guía razonable para el razonamiento práctico. Con este fin procederé del siguiente modo: primero, expondré cómo opera la distinción intentado/colateral y qué situaciones cabe diferenciar conforme a ella en el ámbito que nos ocupa; segundo, responderé al argumento de
Judith Thomson que Figueroa ofrece como prueba concluyente contra la relevancia de la distinción intentado/colateral; tercero, desarrollaré el argumento que permite demostrar que tal distinción sí es moralmente relevante y debe ser recogida por el Derecho.

\section{El sentido del PDE y la distinción intentado/colateral}

El PDE establece que un efecto malo que sería siempre inmoral intentar (esto es, procurar como fin o como medio) puede, no obstante, provocarse justificadamente si sólo se sigue como efecto colateral de una acción en sí misma lícita y necesaria para conseguir un bien de importancia proporcionada. El ejemplo paradigmático de este tipo de efectos es la muerte de un ser humano 
inocente. Quien mata intencionalmente a un inocente, ya sea a un tercero o a uno mismo, comete necesariamente «homicidio» o «suicidio», actos siempre malos o injustos, que están, por lo mismo, absolutamente prohibidos. Por el contrario, quien realiza una acción en sí misma lícita de la que se sigue como efecto colateral previsto la muerte de un inocente, no siempre comete homicidio o suicidio: actúa justificadamente si la acción es necesaria para alcanzar un bien de importancia proporcionada.

Las reglas precedentes también se aplican a la muerte de enfermos graves. Conforme al PDE, la eutanasia y el suicidio médicamente asistido son actos siempre ilícitos, pues en ambos se intenta la muerte del enfermo como medio para poner término a sus sufrimientos. En cambio, según el mismo principio, es lícito suministrar a un enfermo terminal un tratamiento paliativo proporcionado, aun cuando se prevea que, como efecto colateral, acelerará la muerte. Y es igualmente lícito omitir o interrumpir la aplicación de medios o tratamientos desproporcionados, aunque se prevea que de esto se seguirá la muerte del paciente, cuando esa muerte sólo es tolerada como efecto colateral de un acto en el que se intenta evitar dolores innecesarios, gastos desmedidos o uso superfluo de recursos o instalaciones escasos que ya no reportarán beneficio.

Esta aplicación tradicional del PDE ha sido recogida por el Derecho. Ella encuentra su refrendo jurisprudencial en las sentencias de los casos Vacco vs. Quill, dictada por la Corte Suprema de los Estados Unidos de Norteamérica en 1997, y Rodríguez vs. British Columbia, dictada por la Corte Suprema de Canadá en 1993. Además, fue admitida en una enmienda del año 2003 al Código Penal de Queensland.

Vacco vs. Quill se origina cuando un grupo de médicos y enfermos terminales demandan al estado de Nueva York bajo el alegato de que su ley penal violaba la Decimocuarta Enmienda de la Constitución de los Estados Unidos de Norteamérica, que garantiza a toda persona la igual protección de las leyes. Los demandantes afirmaban que la ley estatal establecía una distinción arbitraria al prohibir, por una parte, el suicidio médicamente asistido, y permitir, por la otra, acciones médicas que producen como efecto previsto la muerte del paciente: los referidos tratamientos paliativos y el rechazo o interrupción de tratamientos que permi- tirían mantener al paciente con vida. Al resolver, la Corte deniega la petición de los demandantes con el argumento de que la distinción supuesta en la ley no es arbitraria, sino que, por el contrario, está de acuerdo con los principios jurídicos fundamentales sobre la causalidad y la intención. Y establece: «El Derecho desde siempre ha usado la intención o propósito del agente para distinguir entre dos actos que pueden tener el mismo resultado [...]. Dicho de otro modo, el Derecho distingue entre acciones realizadas "por causa de" ("because of") un determinado fin y acciones realizadas "a pesar de" ("in spite of") sus no intentadas pero previstas consecuencias» (521 U.S. 793 [1997]).

En Rodríguez vs. British Columbia, la demandante alegó que la prohibición penal del suicidio asistido era contraria a diversas disposiciones de la Canadian Charter of Rights and Freedoms. La Corte, al pronunciarse sobre el mismo argumento invocado por los impugnantes de la ley penal de Nueva York, resuelve lo siguiente: «... la distinción trazada aquí se basa en la intención: en el caso del cuidado paliativo la intención es aliviar el dolor, lo que tiene el efecto de acelerar la muerte, mientras que en el caso del suicidio asistido la intención es innegablemente causar la muerte. [...] las distinciones basadas en la intención son importantes, $\mathrm{y}$, de hecho, forman las bases de nuestro Derecho Penal» ([1993] 3 S.C.R. 519).

La sección 282A del Queensland Criminal Code Act 1899 señala que una persona no es criminalmente responsable por proporcionar cuidados paliativos a otra si lo hace con razonable habilidad y cuidado. Y luego agrega que esto «se aplica incluso si un efecto incidental de proporcionar el cuidado paliativo es acelerar la muerte de la otra persona». Pero finalmente añade: «Sin embargo, nada en esta sección autoriza, justifica o excusa (a) un acto u omisión hecho con la intención de matar a otra persona; o (b) ayudar a otra persona a matarse a sí misma».

Esta última cita da ocasión para decir que, conforme al PDE, las distinciones acción/omisión y hacer/permitir carecen de relevancia para determinar especies de acciones. Así, el médico que mata intencionalmente al enfermo para poner fin a sus sufrimientos comete eutanasia sea que lo mate (i) no dándole el medicamento que puede y debe darle para mantenerlo con vida o (ii) dándole una sobredosis de ese medicamento. Comparto, pues, los reparos de Figueroa contra la distinción hacer/ 
permitir, pero agrego que es un error pensar que ella deba ser invocada para justificar la omisión o interrupción de un tratamiento desproporcionado. Esta omisión o interrupción sólo se justifica porque no se efectúa con intención de matar. De hecho, el proyecto de ley que motiva el trabajo de Figueroa no atribuye, en esta materia, relevancia a la distinción hacer/permitir, sino precisamente a la distinción intentado/colateral, como resulta claro de sus actuales artículos 14 y 16, que establecen que el rechazo a tratamientos en ningún caso podrá tener o implicar como objetivo la aceleración artificial de la muerte.

\section{Respuesta al argumento de Thomson}

Figueroa comienza su crítica contra el PDE señalando que «existe considerable doctrina en la filosofía moral inglesa que objeta su utilidad». Enseguida menciona algunos autores que lo habrían rechazado. Es claro, sin embargo, que la sola existencia de detractores del PDE no es un argumento contra su validez. Por lo demás, son muchos también sus partidarios, como Anscombe ${ }^{2}$, Finnis ${ }^{3}$, Boyle $^{4}$, Cavanaugh ${ }^{5}$, Kaczor ${ }^{6}$ o, pace Figueroa, la propia Foot, que terminó por aceptar la relevancia moral de la distinción intentado/colateral ${ }^{7}$. En realidad, Figueroa presenta sólo un argumento contra el PDE. Mas este argumento, que está tomado de Thomson, puede ser fácilmente refutado, pues atribuye al PDE algo que éste no implica.

Según Thomson, el PDE conduce a la absurda conclusión de que no se podría aplicar sedación terminal a un enfermo grave si el único médico disponible fuera un partidario de la eutanasia que, por tanto, inyectaría la droga paliativa con intención de matar. En este caso, piensa ella, el principio obligaría a buscar un médico que administre la droga con intención de aliviar el dolor; y si ese médico no se encuentra, habría que dejar que el paciente continúe sufriendo ${ }^{8}$.

Pero Thomson olvida que el PDE no sólo es compatible con, sino que surge y se inserta armónicamente en, una teoría ética que reconoce que la justificación moral es más exigente que la justificación jurídica ${ }^{9}$. El Derecho, en efecto, versa directamente sobre la justicia o injusticia de los actos externos, y por eso «sólo atiende a la intención en cuanto se revela o incluye en los actos externos, y la supone buena o mala conforme a la naturaleza de las acciones ${ }^{10}$. Pensemos en una mujer recién em- barazada que se somete a una histerectomía para detener un cáncer cervical. Si la mujer realmente padecía cáncer y no era posible diferir la operación para después del parto, entonces, conforme al PDE, esta acción está justificada desde el punto de vista jurídico, pues objetivamente la muerte del feto es sólo un efecto colateral de un acto terapéutico en sí mismo lícito, necesario y proporcionado. Pero ¿qué pasa si el médico que practica la operación desea que muera el feto, porque está enamorado de la mujer y no quiere que tenga hijos de otro hombre? La respuesta es sencilla: aunque este médico tiene una voluntad desordenada, la acción no deja de estar jurídicamente justificada. Algo análogo sucede en el caso de un salvavidas que, frente a dos bañistas (A y B) que se ahogan, opta por salvar a A sabiendo que eso implicará no salvar a B. Si efectivamente era imposible salvar a los dos bañistas porque se ahogaban al mismo tiempo en sectores de la playa distantes entre sí, entonces, conforme al PDE, esta acción está justificada desde el punto de vista jurídico, pues objetivamente el no salvar a $B$ es sólo un efecto colateral de un acto de rescate en sí mismo lícito, necesario y proporcionado. Pero ¿qué pasa si el salvavidas optó por salvar a A porque sentía odio por B? Nuevamente: aunque el salvavidas tiene una mala voluntad, la acción no deja de estar jurídicamente justificada. Pues bien, lo mismo sucede con el caso de Thomson. Es decir, si la sustancia que se administra al enfermo tiene virtudes paliativas ( $v$. gr., es morfina y no cianuro), si la dosis que se le aplica es la exigida por la evolución del dolor según su historia clínica y, en fin, si se cumplen los demás requisitos del caso para que el tratamiento sea necesario y proporcionado, entonces, conforme al PDE, esta acción está justificada desde el punto de vista jurídico, pues objetivamente la aceleración de la muerte será sólo un efecto colateral de un tratamiento paliativo lícito. El médico que se complace en la muerte del enfermo, ya sea porque es partidario de la eutanasia o porque lo odiaba, tiene una mala voluntad, pero actúa justificadamente ante el Derecho, pues de internis non iudicat praetor.

\section{La relevancia moral de la distinción intentado/colateral}

El PDE comprende una faz prohibitiva y una faz permisiva. En efecto, él supone dos tesis morales: (i) que existen ciertos efectos malos que siem- 
pre está prohibido intentar (i.e., procurar como fin o como medio) y (ii) que está permitido provocar esos mismos efectos malos si ellos se siguen como efecto colateral de una acción en sí misma lícita y necesaria para alcanzar un bien de importancia proporcionada. A continuación defenderé las dos vertientes del PDE tomando como modelo el caso del homicidio. Lo que diga de él puede extenderse a la eutanasia (que es una forma de homicidio) $y$, mutatis mutandis, al suicidio asistido.

\subsection{Justificación de la faz prohibitiva del PDE}

La idea de que el homicidio, como acto siempre injusto, consiste en matar intencionalmente a un ser humano inocente -esto es, en proponerse matar a un ser humano inocente ya sea como fin de la acción o como medio para conseguir otro fin-contiene elementos que están fuera de controversia. Así, todos consideran que es injusto proponerse matar a un inocente como fin del acto, o sea, por pura malicia o gozo en el mal ajeno. Del mismo modo, todos aceptan que es injusto matar a un inocente como medio para un mal fin, como cuando alguien mata a un hombre para robarle. En lo que no todos convienen es en que sea siempre injusto matar a un inocente como medio para un buen fin. Algunos piensan, por ejemplo, que es lícito matar a uno como medio para que un terrorista se abstenga de matar a diez, o que es lícito matar al no nacido como medio para salvar a la madre, $o$, en fin, que es lícito matar a un moribundo como medio para que deje de sufrir. Otros adoptan una versión más moderada de esta tesis, y afirman que es lícito matar a un inocente para salvar a otro cuando de lo contrario morirán los dos. Por ejemplo, dicen que es lícito matar al no nacido para salvar a la madre cuando, si no se actúa así, morirán la madre y el feto; o que es lícito matar a un inocente cuando un terrorista nos amenaza con que, de otro modo, él mismo matará a ese inocente y a diez más.

La tesis de que es lícito matar a un inocente como medio para un buen fin es característica de las éticas consecuencialistas. Mas hay buenas razones para rechazar este tipo de doctrinas. En particular, ellas constituyen una negación práctica de la dignidad de la persona humana, pues esta dignidad exige que nunca se trate a un ser humano como si fuera un mero medio, instrumento o $\cos \mathrm{a}^{11}$. Para las teorías consecuencialistas, en cambio, no hay formas de tratar a un ser humano respecto de las cuales se pueda decir que, cualesquiera que sean las consecuencias, nadie debe ser jamás tratado de esa forma ${ }^{12}$. En términos jurídicos, esto significa que las teorías consecuencialistas implican la abolición de los derechos humanos inviolables (que garantizan la referida dignidad). Como dice Finnis: «El principio de que el mal no puede ser hecho por el bien [...] es el fundamento de los derechos humanos verdaderamente inviolables (absolutos) y es la columna vertebral de los sistemas jurídicos decentes. Pues un sistema jurídico decente excluye incondicionalmente la occisión o el daño de personas inocentes como medio para cualquier fin, ya sea público o privado» $»^{13}$. En suma, el consecuencialismo acaba por reducir al hombre a la condición de un mero instrumento y se erige como una teoría moral para la que todo puede estar permitido. Las prohibiciones morales absolutas de la tradición del PDE constituyen, por el contrario, una defensa de la dignidad de la persona, en la medida en que fijan los límites de la conducta humana ${ }^{14}$, límites cuya transgresión degrada al hombre.

\subsection{Justificación de la faz permisiva del PDE}

Frecuentemente se pasa por alto que el PDE no sólo debe defenderse de un consecuencialismo que suprime la dignidad de la persona. En el otro extremo, existe una teoría que rechaza lo que hemos denominado faz permisiva del PDE. Si continuamos con nuestro ejemplo del homicidio, esta teoría vendría a decir que se viola la dignidad humana -esto es, se comete homicidio como acto siempre injusto- no sólo cuando (i) la muerte del inocente es un efecto intentado por el agente, sino también cuando (ii) esa muerte es un efecto colateral previsto de lo que el agente elige.

Una posición que niegue tanto el consecuencialismo como la faz permisiva del PDE sólo podría decir que el homicidio, como acto siempre prohibido, consiste en (a) realizar una acción positiva de la que se sigue previsiblemente la muerte de un ser humano inocente, o en (b) optar por un curso de acción (acción positiva u omisión) del que se sigue previsiblemente la muerte de un ser humano inocente.

Sin embargo, una norma absoluta que prohíba (b) debe descartarse, pues es imposible de cumplir. Así, un médico que sólo puede salvar la vida de la madre con una acción de la que se sigue la muerte del feto, y viceversa, sería necesariamente culpable 
de homicidio, lo que es absurdo. Una prohibición absoluta de (a) no produce tal incoherencia, ya que siempre puede cumplirse mediante la omisión. Con todo, cabe objetar que la distinción acción/ omisión sólo implica diferencias en el orden de la causalidad física, por lo que no hay razones para sostener que un agente, obligado prima facie a evitar dos efectos colaterales malos, esté siempre más obligado a evitar el que resulta de su acción que el que resulta de su omisión, cuando la acción con la que impide uno es la causa del otro. Todo dependerá, aquí, de la razón proporcionada para actuar $^{15}$. En cambio, la distinción intentado/colateral implica diferencias en la voluntariedad, que es de donde fluye la moralidad de los actos humanos, pues los actos se consideran humanos o morales en la medida en que son voluntarios. De ahí, pues, que sea ésta la distinción moralmente relevante para fijar el alcance de una norma moral o jurídica absoluta como la que prohíbe el homicidio.

De paso, el PDE evita el rigorismo que supondría limitar la prohibición absoluta del homicidio en función de la distinción acción/omisión. Este rigorismo impediría efectuar tratamientos paliativos a enfermos terminales, pues exigiría al agente abstenerse de actuar, sin importar cuál sea el bien del que se verá privado, cada vez que prevea que de su acción se seguirá la muerte de un inocente, incluso como efecto colateral.

\section{Conclusiones}

En este trabajo se ofrecen argumentos filosóficos para demostrar la relevancia moral de la distinción entre efectos intentados (queridos como fin o como medio) y efectos colaterales previstos. En síntesis, se muestra que el PDE constituye una suerte de áurea medianía -un justo medio-entre un consecuencialismo que acaba por negar la dignidad de la persona y un causalismo que atribuye una relevancia infundada a los vínculos causales de orden físico en desmedro de las disposiciones volitivas. Todo esto permite concluir que el PDE puede usarse como un criterio válido para distinguir entre verdaderas situaciones de eutanasia o suicidio asistido (donde se mata como medio para un fin) y otras acciones que pueden parecérseles en su estructura física pero difieren de ellas desde el punto de vista moral y jurídico.

\section{Referencias}

1. Figueroa R. Matar, dejar morir y eutanasia en el proyecto de ley sobre derechos de las personas y en la doctrina chilena. Rev Med Chile 2011; 139: 655-59.

2. Anscombe E. War and Murder. En: Stein W, editor, Nuclear Weapons: A Catholic Response, New York: Sheed and Ward 1961; 45-62.

3. Finnis J. Intention and Side Effects. En: Frey R, Morris C, editores, Liability and Responsibility: Essays in Law and Morals, Cambridge: Cambridge University Press, 1991; 32-64.

4. Boyle J. Medical Ethics and Double Effect: The Case of Terminal Sedation.Theor Med Bioeth 2004; 25: 51-60.

5. Cavanaugh T. Double-Effect Reasoning: Doing Good and Avoiding Evil, Oxford: Oxford University Press, 2006.

6. Kaczor C. Faith and Reason and Physician-Assisted Suicide. Christian Bioethics 1998; 4: 183-201.

7. Foot P. Morality, Action, and Outcome. En: Honderich T, editor, Morality and Objectivity: A Tribute to J. L. Mackie, London: Routledge and Kegan Paul, 1985; 23-38, nota 6.

8. Thomson J. Physician-Assisted Suicide: Two Moral Arguments. Ethics 1999; 109: 497-518.

9. Miranda A. El principio del doble efecto y su relevancia en el razonamiento jurídico. Revista Chilena de Derecho 2008; 35: 485-519.

10. Fernández Concha R. Filosofía del derecho o derecho natural, 3. ${ }^{\mathrm{a}}$ ed., Santiago: Editorial Jurídica de Chile, 1966, t. I, 158.

11. Kant I. Grundlegung zur Metaphysik der Sitten, Akademie-Ausgabe IV, Berlin: Walter de Gruyter, 1968; 429: 10-24.

12. Finnis J. Natural Law and Natural Rights, Oxford: Clarendon Press, 1980; 223-6.

13. Finnis J. Commensuration and Public Reason. En: Chang R, editor, Incommensurability, Incomparability, and Practical Reason, Cambridge (Massachusetts): Harvard University Press 1997; 215-33 [226].

14. Tomás de Aquino. Summa theologiae, II-II, q. 79, a. 2, c.

15. Kramer H. The Indirect Voluntary or Voluntarium in Causa, Washington D. C.: The Catholic University of America, 1935; 64-73. 